\title{
Identidad y multiculturalismo. Una perspectiva teórica del pluralismo y reconocimiento para las minorías nacionales
}

\author{
Tristano Volpato ${ }^{1}$
}

Fecha de recepción: 23 de julio de 2013

Fecha de aprobación: 7 de octubre de 2013

\begin{abstract}
Resumen
Con base en la propuesta multicultural contemporánea, el objetivo del artículo es presentar teóricamente algunas dinámicas de negociación de la identidad, tomando en consideración tanto los cambios en el proceso de comunicación y sus efectos sobre la cultura, el habitus, el modus vivendi, como la autopercepción de los actores sociales que componen las minorías nacionales. En primer lugar se discute el rol de la comunicación en el proceso de reconocimiento. En segunda instancia analizamos el concepto de identidad enfatizando la relación entre representación y multiculturalidad, y la idea de identidad múltiple.
\end{abstract}

Palabras clave: comunicación, identidad, reconocimiento.

\begin{abstract}
Based on the contemporary multicultural proposal, the objective of this article is to explore theoretically some dynamics for the negotiation of identity taking into account changes in the communication process and their effects on culture, the habitus, the modus vivendi, and on the self-perceptions of national minorities. It firstly discusses the role of communication in the recognition process, and it, secondly, analyses the identity concept highlighting the relationship between representation and multiculturalism as well as the idea of a multiple identity.
\end{abstract}

Key words: communication, identity, recognition.

1 Maestría en Ciencias Sociales. Investigador del Área de Investigación Aplicada y Opinión del Instituto de Investigaciones Jurídicas de la Universidad Nacional Autónoma de México, Ciudad de México, México.

Dirección postal: Calle Cuautla n. 51-6, C.P. 14420, Col. Santa Ursula Xitla, Del. Tlalpan, Ciudad de México, México.

Dirección electrónica: tristano.volpato@gmail.com, 
Identidad cultural y multiculturalismo. Una perspectiva teórica del pluralismo y reconocimiento para las minorías nacionales - Tristano Volpato

\section{Prefacio}

En el contexto de las sociedades globales reducir la sub-representación y ofrecer parámetros institucionales para una correcta negociación de la identidad individual y colectiva representan los elementos de mayor relevancia para aquellos Estados que los filósofos políticos definen poliétnicos o multinacionales (Kymlicka, 1996, 2002 y 2007; Rawls, 1971; Barry, 2002; Waldron, 2000; Walzer, 1992).

Por contraste, al referirnos al concepto de identidad no es posible definir uniformemente su significado y más bien este varía dependiendo del contexto, de su utilidad social y de la legitimidad que los Estados deciden otorgar o negar a su reconocimiento.

De esa forma, a pesar de que la identidad haya cobrado una importancia imprescindible para las minorías nacionales, sobre todo en la producción de un cierto número de derechos destinados a modificar positivamente las interrelaciones entre subgrupos y Estado (González Manrique, 2006; Falconi, Hercowitz y Muradian, 2004), en términos generales "la realización plena del pluralismo jurídico... continúa siendo una utopía" (Rodrigues Pinto y Domínguez Ávila, 2011: 59; Ariza Santamaría, 2006). Lo cual sugiere que muchos países no hayan interiorizado la utilidad del principio de identidad para la integración y el uso del poder y los recursos o que, simplemente, al interpretar erróneamente el significado del concepto, los Estados no hayan sido lo suficientemente efectivos en el "acomodo" (Kymlicka, 1996; Kymlicka y Norman, 2000) de la diversidad cultural que caracteriza a sus sociedades.

Con el objetivo de analizar las dinámicas del reconocimiento y representación para las minorías nacionales, se afronta así el significado del concepto de identidad en su "modalidad" multicultural y se indagan teóricamente sus propiedades de reconocimiento y autopercepción, tanto en relación a las dinámicas de homogeneización-diversificación cultural de las mino- 
rías, como con respecto a las interrelaciones entre sub-grupos y Estado.

De esa manera no se pretenden teorizar solamente sus elementos característicos, sino también la utilidad práctica del concepto de identidad en los contextos socioculturales plurales actuales.

El trabajo se organiza en tres momentos: en primer lugar se analiza el concepto, tomando en consideración la idea de multiculturalismo en sus modalidades "manejado" y "transformativo"; en segunda instancia se hace referencia a los motivos que llevan a los actores a escoger el grupo de pertenencia, especialmente a partir de los componentes cognitivo, evaluativo y emotivo del proceso de autodeterminación colectiva; finalmente se afronta el concepto de identidad múltiple, como efecto empírico del proceso.

Los resultados del estudio se distribuyen entre la ampliación del significado relativo al concepto de identidad, y un intento de explicar su utilidad práctica en el contexto multicultural de las sociedades contemporáneas.

\section{Comunicación, identidad y multiculturalismo}

El elemento imprescindible para la comprensión del concepto de identidad en el contexto de las sociedades multiculturales es representado por la relación entre minorías y Estado. Una relación que Leydesdorff (2003: 123) considera el producto de una transición a la modernidad que se ha encargado de modificar radicalmente la naturaleza de la comunicación per sé, sobre todo en relación a lo que el autor considera ser las unidades comunicativas responsables de asegurar aquellas manifestaciones de la interacción directa entre los actores sociales y los significados intrínsecos de dichas interrelaciones "con sentido".

En este contexto, la modificación de las formas con las cuales el proceso de comunicación se integra a las dinámicas sociales, 
Identidad cultural y multiculturalismo. Una perspectiva teórica del pluralismo y reconocimiento para las minorías nacionales - Tristano Volpato

culturales y políticas de un cierto universo simbólico condiciona a su vez la tipología de identidad negociable para las minorías y el marco cultural de auto-reconocimiento que caracteriza los miembros de las comunidades involucradas en este proceso.

La importancia de estudiar la identidad individual y colectiva de actores o grupos específicos se justifica así de manera dicotómica. Por un lado, por la utilidad metodológica que deriva de su estudio teórico en el reconocimiento de la diversidad cultural como un valor. Por el otro, por los efectos potencialmente positivos que cobra el conocimiento de las dinámicas de interrelación y representatividad, sobre las costumbres, las tradiciones y la cultura que caracterizan el modus vivendi de los grupos y de sus miembros (Bell y Newby, 1971).

El acercamiento al concepto de identidad y sus implicaciones concretas en la cotidianidad no pueden así obviar una tensión teórico-empírica del proceso de reconocimiento sociocultural de los sub-grupos. Por lo contrario, al mismo tiempo en que implica incluir las necesidades específicas, propiedades, derechos y formas auto definidas de representación de las minorías (Cerulo, 1997: 385-409), supone analizar las implicaciones de la representatividad de grupo y destacar la necesidad de una suerte de acuerdo formal o informal entre minorías e instituciones políticas para la organización de los espacios culturales individuales y colectivos, destinados a generar una dinámica social de respeto e integración (Kymlicka y Norman, 2000; Taylor, 1931: 28, 2000).

Con estas premisas, y en base a las características culturales, sociales, económicas o raciales de los actores que constituyen las minorías nacionales, es posible entonces definir las comunidades tomando en consideración las ideologías de sus miembros, sus necesidades, los objetivos comunes consolidados por la presencia de símbolos, su modus vivendi y sus valores, suponiendo una relación directa con el Estado.

Al considerar estos elementos durante el proceso de reconocimiento, las minorías obtienen la oportunidad de construir sus 
propias formas de representación y autodeterminación, y deciden valorar sus exigencias básicas de integración social, política o económica, o bien crear un mecanismo de autopercepción a partir de lo que Izard Martínez (2005) definió como una suerte de filosofía del retorno. En este contexto, los actores pueden reconocerse como parte de una sociedad más amplia que ofrece un conjunto ecuo de derechos para los sub-grupos y la "cultura dominante", aún sin obviar su posición de actores cuyo reconocimiento mutuo depende de una trayectoria histórica común resultante de un cierto grado de "memoria colectiva".

Así, dependiendo de la forma según la cual las minorías deciden reconocerse y relacionarse con las instituciones y el Estado, o por lo contrario, dependiendo de la predisposición de las instituciones hacia las exigencias de los grupos nacionales, las minorías pueden obtener el reconocimiento de sus derechos específicos, tanto en base a las condiciones culturales, económicas o sociales que las comunidades hacen manifiestas, como a partir de parámetros culturales que el Estado no considera ser limitantes a la conservación de los valores nacionales. Si el Estado acepta la normatividad intergrupal de las comunidades, los grupos obtienen fácilmente lo que los multiculturalistas contemporáneos definen como un proceso social cuyo resultado tomaría el semblante de un pluralismo basado sobre la justicia compartida (Kymlicka y Norman, 2000; De Zwart, 2005; Rawls, 1992)2. Si por lo contrario, el Estado excluye deliberadamente las exigencias de las minorías y obvia la existencia de ciertas normas integrupales de construcción de la identidad comunitaria, los grupos asumen las conse-

2 Impulsar la producción de derechos especiales para las minorías nacionales e imponer un principio de pertenencia cultural para los miembros de las comunidades locales representa la solución política propuesta por los multiculturalistas. Ejemplos de lo mencionado son la "asimilación forzada" (enforced asimilation), resultado de un proceso de equidad y justicia compartida en países asimilacionistas; el "acomodo facilitado" (facilitating assimilation), imprescindible para los Estados multiculturales liberales; el rechazo a lo que los multiculturalistas contemporáneos definen colorblind constitution. 
Identidad cultural y multiculturalismo. Una perspectiva teórica del pluralismo y reconocimiento para las minorías nacionales - Tristano Volpato

cuencias de lo que Assies, haciendo ecos a la reflexión teórica de Hale, define una tensión institucional entre multiculturalismo manejado (managed multiculturalism) y multiculturalismo transformativo (transformative multiculturalism) (Assies, 2005).

"El multiculturalismo manejado celebra el pluralismo cultural pero sin traducirse en efectos concretos y durables para los miembros del grupo cultural oprimido[;]...el multiculturalismo transformativo efectúa una real redistribución del poder y de los recursos" (Assies, 2005: 3). Un modelo éste:

que obvia todos aquellos juicios de valor que limitarían la recuperación legítima de la memoria colectiva y el uso de una específica reflexión heurística a partir de la cual estimar la forma más conveniente de distribuir y "acomodar" los grupos (Messick y Mackie, 1989: 55; trad. del autor).

Los dos modelos, a su vez, corresponderían a un multiculturalismo 'desde arriba' y un multiculturalismo 'desde abajo'. En el primer caso se reforzarían las expresiones esencialistas y limitadas (bounded) de las identidades grupales mientras el segundo caso sería asociado con palabras clave de las políticas identitarias progresistas como son la 'heterogeneidad' y la 'hibrididad' (Assies, 2005: 3).

Así, si consideramos el concepto de identidad en la explicación de los motivos para la aceptación o el rechazo, no nos limitamos a la descripción de las dinámicas de exclusión, potencial hostilidad hacia la comunidad, grado personal de autopercepción, proceso de interiorización normativa. Además tenemos que considerar un cierto grado de construcción sistemática de los prejuicios, de la aceptación, de la comunicatividad intragrupal y de la modificación de los parámetros de reproducción normativa, dependientes de la movilidad social entre minorías (Tajfel, 1981: 131-133, 1982: 56-61). Lo cual supone considerar el proceso a través del cual un actor decide participar en un grupo o, en el caso 
de una comunidad, asumir los mecanismos de condicionamiento entre minorías como una continua tensión entre auto y héteroreconocimiento. Esta relación permite además el análisis de los comportamientos individuales de los actores sociales según su personal evaluación de los valores comunitarios, del nivel de implicación emotiva con las ideas o principios sobre los cuales se construye la cultura de grupo, o el tipo de reconocimiento de identidad que los individuos buscan dentro de un grupo específico (Cerulo, 1997: 385-386).

Si los actores valoran la negociación de un cierto nivel de representatividad creado a partir de un criterio de auto-reconocimiento, estaremos hablando de lo que la psicología social define un proceso de construcción de significados auto-relevantes (Tajfel, 1981). Por lo contrario, si las minorías y sus miembros entran a tomar parte de una sociedad cuya praxis implica la presencia de un multiculturalismo transformativo, el concepto de identidad asumirá el significado de un sistema de estructuras autopoiéticas que permiten la asimilación al sistema cultural generalizado de las pautas de valor minoritarias, sin obviar un proceso de diferenciación entre un universo simbólico localizado y una cultura "dominante".

El Estado se hará entonces promotor tanto de la interiorización de cánones normativos ajenos a los del grupo cultural de origen, como de la creación de un cierto nivel de identidad múltiple para todos los participantes en dicha dinámica.

\section{Significados auto-relevantes e identidad}

Para obtener un cierto conjunto de significados auto-relevantes, los actores emplean tres tipos de componentes psicológicos. El primero, de tipo cognitivo, ayuda a crear una conciencia de grupo basada sobre la percepción de un "otro generalizado" que comparte el mismo espacio de vida, normas y valores de cualquier otro actor que pertenece al ambiente cultural. El segundo, de tipo 
Identidad cultural y multiculturalismo. Una perspectiva teórica del pluralismo y reconocimiento para las minorías nacionales - Tristano Volpato

evaluativo, permite una clasificación del sentido de pertenencia expresando el grado de preferencia para un grupo específico. El tercero, emotivo, explica el grado de participación de los actores al contexto al cual ellos mismos pertenecen (Tajfel, 1978: 27-29).

En el primer caso el actor entiende las dinámicas de pertenencia a partir de una elección individual o de una imposición cultural a priori. Los individuos deciden así participar espontáneamente en un grupo elegido en la base de sus intereses y necesidades, o bien a causa de un background étnico que les impone, de manera directa, pertenecer a un cierto ambiente cultural (Réaume, 2000).

En el segundo, la pertenencia potencial o legítima al grupo tomará en consideración el sentido de ser parte de la comunidad de destino en la base de una elección por necesidad o conveniencia. Una evaluación positiva contribuirá al sentido de pertenencia en términos de "membresía formal"; si negativa, esta implicará un sentimiento de autopercepción hacia la amplificación de una dinámica de diferenciación entre miembros y grupo, o entre comunidades culturales, con la sociedad en su conjunto (Deschamps y Doise, 1978; Dutton, Dukerich y Harquail, 1994).

El componente emotivo explica finalmente el grado de conexión de un actor social y su compromiso potencial con la comunidad de pertenencia. En este caso, la conexión emotiva con la historia, la etnia o la raza -aquí entendida como diferencia estética (Wade, 1997, 2005; Wieviorka, 1992; Skidmore y Graham, 1990; Jennings, 1946; Smedley, 1998; Douglas, 1970; Jones, 2005; Taylor, 2000) - alimenta el proceso de absorción del sentimiento de pertenencia per se y condiciona a los actores a tomar parte de un proceso dicotómico de construcción de la identidad: por una parte, mientras su identidad será condicionada por el habitus, el modus vivendi, las creencias y el background cultural, los individuos modificarán (temporal o permanentemente) su propia identidad individual. Por la otra, los actores construirán nuevos parámetros de representación que podrán ser mantenidos, modificados o removidos del contexto cultural en el cual habrán 
sido producidos o hacia el cual habrán sido traídos ${ }^{3}$. Dichos nuevos parámetros vendrán reconocidos como válidos y extensibles a todos los miembros del grupo y empezarán a encarnar la forma según la cual los individuos que tomarán parte de la nueva comunidad demostrarán haber interiorizado ciertas cualidades humanas, sociales, culturales, lingüísticas, religiosas, u otras. Estas características contribuirán a la creación de lo que definimos como identidad colectiva.

Los actores obtendrán entonces la oportunidad de evaluar la identidad de grupo -ese marco de reconocimiento cultural colectivo basado sobre la suma de las auto-identidades de los actores que participan en este proceso (Giménez, 1997a, 2010; Foreman y Whetten, 2002: 619)- y pondrán en confrontación sus percepciones individuales acerca de su propia identidad con la que los demás miembros quisieran que la identidad del grupo fuera (Reger et al., 1994: 565-584; Foreman y Whetten, 2002; Ashforth y Mael, 1989). Los individuos tendrán además la posibilidad de aprobar un número indefinido de semejanzas entre sí y los demás miembros de la comunidad y serán llevados a crear roles sociales ad hoc que legitimarán el mantenimiento de las tradiciones y las normas de las culturas minoritarias, destacando sólo ciertos atributos culturales (relativamente estables en el tiempo), compartidos por la comunidad de pertenencia.

Creando un proceso de continuidad cultural dentro de las minorías, dicha dinámica contribuirá a la homogeneización de la cultura del grupo. Por contraste, estableciendo nuevos parámetros de reconocimiento para los actores y la comunidad, será garantizada una forma de construcción de la identidad abocada a definir el concepto como una cualidad humana basada sobre un proceso de diferenciación, inter y extra grupal, y finalizado con la creación de percepciones heterogéneas del ser, comparti-

3 Esto proceso se denomina "transculturación" y está ampliamente explicado en la obra de Fernando Ortiz, a la cual se remite para más detalles. 
Identidad cultural y multiculturalismo. Una perspectiva teórica del pluralismo y reconocimiento para las minorías nacionales - Tristano Volpato

das dentro de las minorías, y al mismo tiempo, parte del universo simbólico generalizado (Waldron, 2000).

Ahora bien, si dicho reconocimiento define la identidad individual de quien pertenece a una comunidad específica, por otra parte, este proceso contribuye a establecer una nueva forma de representación colectiva que, definida por Giménez como exoidentidad (Giménez, 2010), encarna el resultado del reconocimiento de ciertos parámetros de reproducción cultural destinados a dar vida a una forma de autopercepción que incrementa la cohesión intergrupal y favorece la generación de parámetros sociales interiorizados.

La importancia de la identidad, individual o colectiva, asume así dos dimensiones complementarias. Por un lado, quien busca el reconocimiento, al mismo tiempo busca formalizar su propia presencia, en un momento histórico definido y en un territorio específico. Por el otro, si un ego viene reconocido por un alter generalizado, individual o colectivo, esto se verifica en razón de un proceso de semejanza o diversidad (Tajfel, 1984: 380-382). En el primer caso se trata de una auto-identificación basada sobre el reconocimiento de nuevos parámetros culturales como parte del patrimonio tradicional de la comunidad. En el segundo, la diversidad representa el elemento imprescindible para definir la presencia de un nuevo universo simbólico que se caracteriza por una cultura propia y por tradiciones ancestrales (como una lengua autóctona), y que se encarga de garantizar o rechazar el reconocimiento institucional de una cierta minoría. Si su reconocimiento vendrá legitimado por el Estado, el grupo incrementará su homogeneidad y colaboración interna (Melucci, 1985; Habermas, 1981). Si la presencia del grupo será obviada, los actores que le pertenecen tenderán a la cohesión y al enfrentamiento (Melucci, 1985; Pallí, 2003: 315-318).

Las comunidades empezarán entonces a reconocerse por sus similitudes con "los demás", aún sin dejar de mostrar la pretensión de ser diferenciadas por características específicas, 
y elegirán una particular sensación de poder mirar a su proprio self a través de los ojos de los demás y percibir su múltiple esencia. Será esta una forma peculiar de sentirse parte de y conciliar la doble identidad garantizada por la autopercepción y la asunción de ser definido por el Estado como un elemento más, formalmente institucionalizado, del contexto sociocultural local (Owens Moore, 2005; Du Bois, 1903; Scollon y Scollon, 1995; Simmel, 1890).

Cuando la identidad sea activada, el individuo percibirá los significados implícitos del self, cuyos resultados se concretarán en una conexión entre identidad y comportamiento. Conexión que, basada sobre un cierto bagaje de significados compartidos, impondrá a los actores asumir comportamientos abocados a la creación de significados correspondientes a los de su identidad estándar (Burke, 2006). Las percepciones de estos significados auto-relevantes se insertarán sobre un mecanismo de comparación que permitirá confrontar con el self las percepciones derivadas de los significados auto-relevantes, e impulsará el factor de distinción hacia la producción de un cierto grado de discrepancia entre las señas comunicativas de la identidad estándar y las normas producidas por la nueva situación.

Finalmente, dicha discrepancia resultará en una falta de correspondencia entre los significados de la norma de identidad y los significados de la situación con que los actores se enfrentan, impulsando, en última instancia, la producción de un cierto grado de "identidad múltiple" (Burke, 2006: 82; trad. e interp. del autor).

\section{Identidad múltiple y sentido de pertenencia}

En los Estados multiculturales, el proceso de negociación de la identidad es dicotómico.

Por una parte involucra un "problema de verificación" de la identidad particular; por la otra impulsa la creación de un 
Identidad cultural y multiculturalismo. Una perspectiva teórica del pluralismo y reconocimiento para las minorías nacionales - Tristano Volpato

número indefinido de identidades múltiples activadas contemporáneamente (Burke, 2006: 84-86; Foreman y Whetten, 2002).

En relación a lo primero, la base del conflicto representa una limitante a la percepción de significados intrínsecos a la situación externa al grupo de origen, y provoca una percepción de significados extra-comunitarios y discrepantes con los que caracterizan la norma de la identidad interna.

En segunda instancia, la referencia es la presencia de un conflicto inter comunitario que se manifiesta cuando dos identidades controlan, al mismo tiempo y en distintos niveles, la misma dimensión de significado.

En este contexto, los significados de las normas de identidad se vuelven más sensibles a los cambios de los estándares de reproducción cultural e, impulsando la identidad hacia su propio cambio, le imponen un proceso específico de verificación (Burke, 2006: 85; trad. e interp. del autor).

El sentido de pertenencia garantiza así la modificación de los parámetros culturales que caracterizan a los actores sociales, sin provocar la pérdida de su identidad estándar. Dicho proceso garantiza además un reconocimiento recíproco que, por un lado, contribuye a la generación de una semejanza sociocultural entre minorías y sociedad civil, y por el otro está abocado a reforzar el principio de diferencia entre cultura dominante y sub-grupos (Scollon y Scollon, 1995: 5-15).

En el primer caso, se genera un proceso de continuidad que alimenta los sentimientos internos a la comunidad, que está dirigido a reforzar la unidad local, y que impulsa la existencia formal de la minoría. De esta manera los miembros del grupo perpetran o modifican su background cultural en base a las expectativas de los que son parte del mismo ambiente social y crean una interacción que sobrentiende la existencia de cánones sociales y cultu- 
rales comunes, interiorizados y mutuamente predecibles (Mead, 1934: $173-178)^{4}$.

En el segundo, los parámetros de reconocimiento mutuo pueden ser dirigidos a definir los actores sociales, bien a partir de un reconocimiento ad intra - ad extra, abocado a la reconstrucción de las dinámicas de diferenciación entre contexto comunitario y nacional, o bien en base al grado en que la sociedad accede a modificar su identidad colectiva, asimilando la cultura de las minorías nacionales (Tessarolo, 1990: 142-145). Este proceso implica una dinámica de pertenencia - diversidad entre actores y ambiente social, e impone obviar todos aquellos juicios de valor que se basan en una caracterización cualitativa de cualquier otro miembro del grupo (Messick y Mackie, 1989: 45-81).

Por otra parte, los parámetros de reconocimiento comunitario destacan la diferencia entre el contexto social de las comunidades y el de la cultura nacional, re-contextualizando la imagen de grupo relacionada con la construcción de una representatividad del otro generalizado, distinto y separado del mundo de la vida, cuyo espacio simbólico idealizado hace referencia a la minoría (Parsons y Shils, 1959; Parsons, 1968). En este caso, el concepto de identidad se basa en dos diversos modelos. Por un lado supone la idea de proceso, una suerte de construcción social apriorísticamente abocada a la producción de una autopercepción "en evolución", resultante de un significado semiótico de la identidad

4 Mead afirma que la diferencia que existe entre la reacción del self, debida a un estímulo impuesto por una acción dirigida hacia un cierto actor social $(I)$ y la respuesta de quien ha reaccionado a ese estímulo (me), se asimila a la dinámica de interacción entre individuos y grupos. Dentro de una minoría, un actor representa así un cierto tipo de me, auto-percibido como un elemento que pertenece a un ambiente social definido e influenciado por el mismo en sus actitudes, pensamientos y comportamientos. Por lo contrario, afuera del grupo de pertenencia o en relación con un segundo conjunto, el individuo es llevado a elegir la forma a través de la cual mostrar sus propios parámetros de representación y generar una vía de comunicación que se transforma en una construcción social específica, denominada identidad. 
Identidad cultural y multiculturalismo. Una perspectiva teórica del pluralismo y reconocimiento para las minorías nacionales - Tristano Volpato

cultural (Geertz, 1992), basada sobre la diferenciación de parámetros de comunicación intergrupal, y caracterizada por dinámicas históricas, en cuanto a construcción del sentido de pertenencia, imprescindibles (Giménez, 1997b). Por el otro, permite explicar la diversidad a partir de una dicotomía praxis-estructura y tiene en cuenta la necesidad de delinear parámetros culturales y sociales definidos, a pesar de las diferencias entre cultura dominante y sub-grupos.

A partir de estas premisas, la identidad de grupo se vuelve institucionalizada y quien es parte de una cierta minoría puede finalmente entrar en contacto con la sociedad civil como "miembro efectivo" y expresar una suerte de ciudadanía localizada que permite a las minorías ser consideradas como espacios culturales liminales entre lo que definiríamos "conciencia nacional", y la formalización de una presencia cultural externa al contexto generalizado, construida a partir de un proceso de legitimación de facto de los derechos de la minoría (Mansbridge, 2000: 99-123).

Al permitir una dinámica de reconocimiento dirigida a crear un cierto nivel de equidad entre minorías y cultura dominante, la identidad impulsa una dinámica de fragmentación de los canales comunicativos entre el actor y los demás miembros de la minoría, y permite una nueva formación de la personalidad.

Misma que no se dirige necesariamente a quien es parte del mismo contexto social, sino hacia una forma de representatividad menos perfectible que incorpora un cierto nivel de reconocimiento sociocultural también para los actores que no son parte del grupo (Simmel, 1890: 54; trad. del autor).

Lo cual, si por un lado, afecta negativamente el sentido de pertenencia y la respuesta institucional a su presencia y reconocimiento, por el otro, garantiza la existencia de parámetros socioculturales diversos que, a pesar de ser parte del universo simbólico generalizado, permiten a las minorías autodefinirse como sub-grupos locales distintos de la cultura dominante (Burke, 
2006: 81-96; Volpato, 2012; French y Weis, 2000; Lamont y Favor, 2007).

La construcción de la identidad colectiva se ve así condicionada no solamente por la naturaleza cultural que subyace al proceso de autodeterminación identitaria (Simmel, 1971); por lo contrario, dicho proceso toma la forma de un parámetro "objetivado" de la cultura local, superpuesto a la realidad de las comunidades y externo a la naturaleza misma de la minoría (Simmel, 1971).

Si se considera la identidad como una forma de reconocimiento mutuo invariable que caracteriza a un grupo de manera indistinta y homogénea, se hablará entonces de una idea de identidad asociada a un principio de igualdad; por lo contrario, al ser construida sobre lo que Giménez define un "criterio de distinción" (Giménez, 1997b), la identidad será asociada a una idea de diferencia que podrá llegar a obviar el rol del ambiente social (Larraín, 2000: 24; trad. e interp. del autor) a través de específicas acciones con sentido.

Dichas acciones tendrán la función de crear la oportunidad de concurrir al reconocimiento institucional de las minorías, aun permitiendo el aislamiento de ciertos grupos a causa de su estética racial, el idioma, la afiliación religiosa, el origen nacional o social, el linaje, el lugar de residencia (Weber, 2001: 82; trad. del autor).

Debido a ello, el actor interactuará con el grupo en el cual toma parte y establecerá una serie de estímulos basados sobre un reconocimiento recíproco y bidireccional.

El primero permitirá identificar el self como un sujeto dotado de características únicas que demuestran su background cultural, su forma de comportarse o su habitus.

El segundo buscará crear una relación sociocultural entre micro-universo simbólico y universo simbólico de referencia, estableciendo un cierto tipo de identidad, elegida por los individuos 
Identidad cultural y multiculturalismo. Una perspectiva teórica del pluralismo y reconocimiento para las minorías nacionales - Tristano Volpato

o los grupos indiferentemente. Identidad resultante del conjunto de cualidades con que una persona o comunidad se perciben íntimamente conectados (Larraín, 2000: 25; trad. del autor).

La dinámica que genera este tipo de identidad puede ser analizada a partir de una perspectiva triple: en base a cómo los individuos se definen a sí mismos frente al marco cultural generalizado (auto-comunicación); a la relación entre individuos y objetos materiales (e inmateriales) que alimentan la conciencia comunitaria (comunicación indirecta); la confrontación entre la percepción de sí mismos -lo que Larraín (2000) define como selfimage - y el reconocimiento del otro (others-recognition). En este caso los esfuerzos comunitarios serán dirigidos a la obtención de un beneficio común, construido a partir de un reconocimiento cultural que busca la institucionalización y la representación del grupo (Habermas, 1981; Melucci, 1985: 150-163).

En primer lugar, el actor y la comunidad cultural se reconocerán recíprocamente generando una dinámica social que les permite percibirse mutuamente y aceptar modelos culturales también alejados de los propios.

En segunda instancia, a partir de un proceso de apropiación de los objetos culturales intercomunitarios, los individuos desarrollarán su sentido de pertenencia y se asumirán mutuamente como miembros formales del mismo grupo.

En este caso, a medida que el contrato social entre grupo y ambiente social incrementará, aumentará también el número de elementos de similitud intergrupal y las características en base a las cuales los actores diferirán, impulsando la variabilidad y la diferenciación percibida (Messick y Mackie, 1989: 55; trad. del autor).

Características que si compartidas, contribuirán al proceso de aceptación y homogeneidad; si contrastantes, provocarán una dinámica de rechazo y discriminación (Link y Phelan, 2001: 363$385,365)$. 
En tercer lugar, a través de una relación ego-alter, la identidad colectiva se institucionaliza y pasa a definir un proceso de evaluación de las condiciones de equidad para la creación colectiva de condiciones sociales, culturales, políticas o económicas abocadas a una formalización de la necesidad de institucionalizar ciertos derechos de minoría (Rawls, 1971: 92; Waldron, 2000). De esa forma, el contenido relacional intercambiado dentro de un cierto grupo cultural representará tanto los atributos mismos del aparato comunicativo de los actores involucrados en esta dinámica, como el canal de comunicación para el desarrollo de una relación exclusiva e interna al grupo (Barry y Crant, 2000: 648-664, 651).

El actor no será así diverso de todos los demás sólo por definición. Por lo contrario, cada individuo destacará por las performances individuales que les permitirán producir un cierto número de significados socioculturales relacionados con el grado de pertenencia percibida, con el nivel de aceptación individual y colectiva, con el grado de diferencia entre los actores involucrados en dicha dinámica (Tuan, 2002: 307-318).

El grupo será entonces el artífice de su misma identidad y asumirá el rol de un proceso intersubjetivo de reconocimiento mutuo que ofrecerá a sus miembros una identidad individual y un conjunto de códigos culturales, potencialmente estandarizados y extensibles a todos los miembros (Larraín, 2000: 27; trad. e interp. del autor).

Dichos códigos permitirán además llevar a cabo parangones cognitivos socialmente relevantes entre cultura local y nacional, categorizando las comunidades y sus características peculiares (Foreman y Whetten, 2002: 618-635).

Mientras los actores buscarán crear un canal de interrelación entre sí mismos y los demás miembros de la comunidad, el principio de diferencia pasará a través de una instrumentalización de la cultura de grupo y generará una suerte de evaluación común del sentido de pertenencia, del reconocimiento recíproco y de la 
Identidad cultural y multiculturalismo. Una perspectiva teórica del pluralismo y reconocimiento para las minorías nacionales - Tristano Volpato

cohesión intergrupal. Dicha relación tenderá a reforzar la identidad individual o colectiva de las comunidades e impulsará la formalización de las normas y de los valores, creando un sentido de significados compartidos y valorizando el sentido de pertenencia (Wiesenfeld, Raghuram y Garud, 1999: 777-790).

Finalmente, si por un lado, la dinámica de reconocimiento y la lucha para la obtención de los derechos de las minorías contribuirán a la definición de aspectos formales del criterio de distinción, por el otro, el efecto de dichas dinámicas establecerá los criterios de reconocimiento para la organización de las diversas identidades y necesidades de los grupos reconocidos por los Estados (Kymlicka y Norman, 2000), y pondrá en marcha un proceso dicotómico de "sistematización negociada" para las minorías nacionales.

Por una parte, ofrecerá a los grupos la posibilidad de participar en la vida social de manera que las perspectivas de paz e integración no se vean disminuidas (Waldron, 1984, 2000: 155; trad. del autor).

Por la otra, impulsará el Estado a brindar la justa atención a los intereses, deseos, y opinión de todos los actores sociales (Waldron, 2000: 155; trad. del autor), ofreciéndoles la oportunidad de gozar de aquellas normas, valores y derechos que, construidos sobre los principios de la libertad, justicia compartida y convivencia, les garantizará un nuevo status social cuyo reconocimiento implicará su integración formal en la definición de la ciudadanía.

\section{Conclusiones}

Estudiar el problema de la negociación de la identidad, especialmente en el contexto de las sociedades multiculturales, implica tener en consideración por lo menos dos aspectos del problema: la forma a través de la cual los grupos buscan el reconocimiento; y su viabilidad dentro del universo simbólico nacional al cual las minorías están adscritas. 
De esa manera, la dinámica de negociación no se aboca solamente a construir un criterio "objetivo" de reconocimiento, sino también a comprender los mecanismos según los cuales se llevan a cabo las relaciones inter y extra comunitarias. Esto garantiza una mejor comprensión también de las vías institucionales para que el Estado asegure la resolución de las exigencias de sus ciudadanos, y garantice la perduración de los valores que caracterizan el territorio en el cual viven dichas minorías.

Con el objetivo de analizar algunos de los procesos de construcción de la identidad de los sub-grupos culturales nacionales, se ha afrontado el tema empleando una perspectiva estrictamente teórica, construida a partir de tres elementos complementarios: la relación entre comunicación, identidad y multiculturalismo; la importancia de los significados auto-relevantes para la creación y comprensión de la identidad de las minorías; el efecto de dichas dinámicas sobre la construcción del sentido de pertenencia y la existencia de una identidad múltiple.

Los resultados de nuestra discusión nos llevan a reflexionar acerca de la existencia de canales de comunicación plúrimos que se articulan entre la predisposición estatal al reconocimiento y los motivos que llevan las minorías a elegir uno u otro parámetro cultural (sea ello la lengua, la estética o las tradiciones) dentro de una tipología específica de multiculturalismo. Si el Estado decide obviar la formalización de la diversidad se hablará de un multiculturalismo manejado, dirigido a reconocer el pluralismo cultural sin traducirse en efectos concretos para las minorías. Por lo contrario, al celebrarse una redistribución del poder y de los recursos, estaremos frente a un multiculturalismo de tipo transformativo. En este contexto los grupos deciden si reconocerse mutuamente a partir de un criterio de similitud -un marco cultural homogéneo que define las minorías por su unidad y cohesión- o en base a un criterio de diversidad, demarcando el límite con la cultura dominante. Dicha separación llevará a escoger la pertenencia en base a criterios empáticos (en donde la afiliación 
Identidad cultural y multiculturalismo. Una perspectiva teórica del pluralismo y reconocimiento para las minorías nacionales - Tristano Volpato

garantizará una membresía informal aunque duradera y concreta), cognitivos (basados sobre una elección racional que se produce por motivos de conveniencia), evaluativos (gracias a los cuales los individuos participan a un grupo elegido en la base de sus intereses o a causa de una "obligación étnica").

Dependiendo de la elección individual efectuada por los miembros de las minorías se delinean las características que transforman los actores en elementos imprescindibles de la existencia misma de los grupos. Al escoger el universo simbólico de pertenencia, se genera una interrelación entre minorías y sociedad civil que crea un cierto parámetro de reproducción normativa resultante de los procesos de modificación cultural mutua: la identidad múltiple.

Finalmente, la discusión propuesta nos lleva a entender el concepto de identidad según dos modalidades distintas y complementarias: por una parte, como un sistema de valores compartido y construido gracias a la interrelación entre minorías y Estado; por la otra, como el elemento imprescindible para la creación de nuevos canales de comunicación y diálogo.

\section{Bibliografía}

Ariza Santamaría, Rosembert (2006): “Democracia con o sin pluralismo jurídico en América Latina". En: Cuadernos de Filosofía Latinoamericana, Vol. 27, n. 94, pp. 74-80.

Ashforth, Blake E. y Mael, Fred A. (1989): "Social Identity Theory and the Organization". En: Academy of Management Review, n. 14, pp. 20-39.

Assies, Willem (2005): “El multiculturalismo latinoamericano al inicio del siglo XXI". Ponencia presentada al evento Pueblos Indígenas de América Latina, Panel 3: "Estados multiétnicos y multiculturales", Programa de Cooperación Internacional de Obra Social-Fundación "La Caixa", Barcelona, 27 y 28 de abril.

Barry, Brian (2002): Culture and Equality. Cambridge, Harvard University Press. 
Barry, Bruce y Crant, Michael J. (2000): “Dyadic Communication Relationship in Organizations: an Attribution/Expectancy Approach. En: Organization Science, Vol. 11, n. 6, Nov.-Dic., pp. 648-664; http:/ / www.jstor.org/stable/2640375

Bell, Colin y Newby, Howard (1971): Community Studies. An Introduction to the Sociology of the Local Community. London, George Allen un Unwin LTD.

Burke, Peter. J. (2006): “Identity Change". En: Social Psychology Quarterly, Vol. 69, n. 1, Marzo, pp. 81-96; http:/ / www.jstor.org/ stable/20141729

Cerulo, Karen A. (1997): "Identity Construction: new Issues, new Directions". En: Annual Review of Sociology, n. 23, pp. 385-409.

Deschamps, Jean C. y Doise, Willem (1978): “Crossed category membership in intergroup relations". En: Differentiation between Social Groups, Henri, Tajfel. London, Academic Press Inc., pp. 141-158.

Douglas, Mary (1970): Pureza y peligro. Un análisis de los conceptos de contaminación y tabú. Madrid, Siglo XXI.

Du Bois, William E.B. (1903): The Souls of Black Folk. Chicago, A.C. Mc Clurg \& Co.

Dutton, Jane E.; Dukerich, Janet M. y Harquail, Celia V. (1994): “Organizational Image and Member Identification". En Administrative Science Quarterly, n. 39, pp. 239-263.

Falconí Fander, Hercowitz Marcelo y Muradian Roldan (2004): Globalización y desarrollo en América Latina. Quito, Flacso Ecuador.

Foreman, Peter y Whetten, David A. (2002): “Members' Identification with Multiple-Identity Organizations". En: Organization Science, Vol. 13, n. 6, Nov.-Dic., pp. 618-635; http://www. jstor.org/stable/3086084

French, Warren y Weis, Alexander (2000): "An Ethics of Care or an Ethics of Justice". En: Journal of Business Ethics, Vol. 27, n. 1/2, pp. 125-136; http:/ / www.jstor.org/stable/25074369

Geertz, Clifford (1992): La interpretación de las culturas. Barcelona, Gedisa.

Giménez, Gilberto (1997a): Cultura e identidades. México, Isunam.

Giménez, Gilberto (1997b): “Materiales para una teoría de las identidades sociales". En: Frontera norte, Instituto de Investigaciones Sociales de la UNAM, México D.F., Vol. 9, n. 18, pp. 9-28. 
Identidad cultural y multiculturalismo. Una perspectiva teórica del pluralismo y reconocimiento para las minorías nacionales - Tristano Volpato

Giménez, Gilberto (2010): “La cultura como identidad y la identidad como cultura". Instituto de Investigaciones Sociales de la UNAM; http://sic.conaculta.gob.mx/documentos/834. doc

González Manrique, Luis Esteban (2006): “QQuiénes somos?: Multiculturalismo y relaciones interétnicas en América Latina". En: Ómnibus, Vol. 12, n. 3; http://www.omni-bus.com/

Habermas, Jürgen (1981): Theorie des kommunikativen Handelns. Band I: Handlungsrationalität und gesellschaftliche Rationalisierung. Band. 2: Zur Kritik der funktionalistischen Vernunft. Frankfurt a. M., Suhrkamp.

Izard Martínez, Gabriel (2005): "Herencia, territorio e identidad en la diáspora africana: hacia una etnografía del retorno". En: Estudios de Asia y África, n. 40, pp. 89-115.

Jennings, H.S., et al. (1946): Aspectos científicos del problema racial. Buenos Aires, Editorial Losada S.A.

Jones, Richard A. (2005): "Race and Revisability". En: Journal of Black Studies, Vol. 35, pp. 612-632.

Kymlicka, Will (1996): Ciudadanía multicultural. Barcelona, Paidós.

Kymlicka, Will (2002): Contemporary Political Philosophy. New York, Oxford University Press.

Kymlicka, Will (2007): Multicultural Odysseys: Navigating the New International Politics of Diversity. New York, Oxford University Press.

Kymlicka, Will y Norman, Wayne (2000): “Citizenship in Culturally Diverse Society: Issues, Contexts, Concepts". En: Citizenship in Diverse Societies, Will, Kymlicka y Wayne, Norman (eds.). Oxford, Oxford University Press, pp. 1-41.

Lamont, Julian y Favor, Christi (2007): "Distributive Justice". En: The Stanford Encyclopedia of Philosophy, Edward N., Zalta (ed.); http:/ / plato.stanford.edu/entries/justice-distributive/

Larraín, Jorge (2000): Identity and Modernity in Latin America. Cambridge, Polity Press.

Leydesdorff, Loet (2003): A Sociological Theory of Communication. The Self-Organization of the Knowledge-Based Society. uPUBLISH. COM.: Universal Publishers; http://www.universal-publishers.com/book.php?method=ISBN\&book=1581126956 
Link, Bruce G. y Phelan, Jo C. (2001): "Conceptualizing Stigma". En: Annual Review of Sociology, n. 27, pp. 363-385.

Mansbridge, Jane (2000): “What does a Representative do? Descriptive Representation in Communicative Settings of Distrust, Uncrystallized Interests, and Historically Denigrated Status". En: Citizenship in Diverse Societies, Will, Kymlicka y Wayne, Norman (comps.), Oxford, Oxford University Press, pp. 99-123.

Mead, George H. (1934): Mind, Self and Society. From the Standpoint of a Social Behaviorist. Chicago, The University of Chicago Press.

Melucci, Alberto (1985): "'Identità e azione collettiva". En: Complessità sociale e identità, Laura, Balbo (et al.). Milano, Franco Angeli, pp. 150-163.

Messick, David M. y Mackie, Diane M. (1989): "Intergroup Relations”. En: Annual Review of Psychology (Deutsche Forschungsgemeinschaft), n. 40; https:/ / www.annualreviews.org/

Owens Moore, Tehodore (2005): “A Fanonian Perspective on Double Consciousness". En: Journal of Black Studies, Vol. 35, n. 6, pp. 751-762.

Pallí, Cristina (2003): "Communities in Context: Undefinition, Multiplicity and Cultural Difference". En: Interamerican Journal of Psychology, Vol. 37, n. 2, pp. 309-326.

Parsons, Talcott (1968): La sociedad. Perspectivas evolutivas y comparativas. México, Editorial Trillas.

Parsons, Talcott y Shils, Edward (edit.) (1959): Toward a General Theory of Action. Massachusetts, Harvard University Press.

Rawls, John (1971): A Theory of Justice. London, Oxford University Press.

Rawls, John (1992): “Justice as Fairness: Political not Metaphysical”. En: Communitarianism and Individualism, Shlomo, Avineri, y Avner, De-Shalit (eds.). Oxford, Oxford University Press, pp. 186-204.

Réaume, Denise G. (2000): “Official-Language Rights: Intrinsic Value and the Protection of Difference". En: Citizenship in Diverse Societies, Will, Kymlicka y Wayne, Norman (eds.). Oxford, Oxford University Press, pp. 245-272.

Reger, Rhonda K.; Gustafson, Loren T.; De Marie, Samuel M. y Mullane, John V. (1994): "Reframing the Organization: why Implementing total Quality is Easier said than Done". En: Academy of Management Review, n. 19, pp. 565-584. 
Identidad cultural y multiculturalismo. Una perspectiva teórica del pluralismo y reconocimiento para las minorías nacionales - Tristano Volpato

Rodrigues Pinto, Simone y Domínguez Ávila, Carlos Federico (2011): "Sociedades plurales, multiculturalismo y derechos indígenas en América Latina". En: Política y Cultura, n. 35, pp. 49-66.

Scollon, Ron y Scollon, Suzanne (1995): Intercultural Communication. Cambridge, Mass. - Oxford, Blackwell Publishers.

Simmel, Georg (1890): Über soziale Differenzierung. Soziologische und psychologische Untersuchungen. Leipzig, Verlag von Duncker und Humblot.

Simmel, Georg (1971): On Individuality and Social Forms. Chicago, University of Chicago Press.

Skidmore, Thomas Elliott y Graham, Richard (1990): The Idea of Race in Latin America, 1870-1940. Austin, University of Texas Press.

Smedley, Audrey (1998): "Race' and the Construction of Human Identity". En: American Anthropologist, Vol. 100, 3, pp. 690-702.

Tajfel, Henri (1978): “Interindividual Behaviour and Intergroup Behavior". En: Differentiation between Social Groups, Henri, Tajfel. London, Academic Press Inc.

Tajfel, Henri (1981): Human Groups and Social Categories: Studies in Social Psychology. Cambridge, Cambridge University Press.

Tajfel, Henri (1982): Gruppenkonflikt und Vorurteil. Bern, Verlag Hans Huber.

Tajfel, Henri (1984): The Social Dimension - vol. II. Cambridge, Cambridge University Press.

Taylor, Charles (1931): Multiculturalism and "the Politics of Recognition". Princeton, Princeton University Press.

Taylor, Paul C. (2000): “Appiah's Uncompleted Argument: W.E.B. Du Bois and the Reality of Race". En: Social Theory and Practice, Vol. 26, n. 1, pp. 103-128.

Tessarolo, Mariselda (1990): Minoranze linguistiche e immagine della lingua. Una ricerca sulla realtà italiana. Milano, Franco Angeli.

Tuan, Yi-Fu (2002): "Community, Society, and the Individual". En: Geographical Review, Vol.92,n.3, Julio, pp.307-318; http:/ /www. jstor.org/stable/pdfplus/4140912.pdf?acceptTC $=$ true

Volpato, Tristano (2012): "Para una teorización del concepto de multiculturalismo latinoamericano". En: Visioni Latinoa- 
mericane, n. 7, pp. 7-29; http://www2.units.it/csal/ home/?file=visioni_latinoamericane_archivio.htm

Wade, Peter (1997): Race and Ethnicity in Latin America. London, Pluto Press.

Wade, Peter (2005): "Rethinking 'Mestizaje': Ideology and Lived Experience". En: Journal of Latin American Studies, Vol. 37, n. 2, pp. 239-257.

Waldron, Jeremy (1984): Theories of Rights. Oxford, Oxford University Press, Oxford.

Waldron, Jeremy (2000): "Cultural Identity and Civic Responsability". En: Citizenship in Diverse Societies, Will, Kymlicka y Wayne, Norman (eds.). New York, Oxford University Press, pp. 155-174.

Walzer, Michael (1992): "Membership". En: Communitarianism and Individualism, Shlomo, Avineri y Avner, De-Shalit (eds.). Oxford, Oxford University Press, pp. 65-84.

Weber, Max (2001): Wirtschaft und Gesellschaft. Gemeinschaften. Tübingen, J.C.B.Mohr-Paul Siebeck [1961].

Wiesenfeld, Batia M.; Raghuram, Sumita y Garud, Raghu (1999): “Communication Patterns as Determinants of Organizational Identification in a Virtual Organization". En: Organization Science, Vol. 10, n. 6, Special Issue: Communication Processes for Virtual Organizations, pp. 777-790; http://www. jstor.org/stable/2640241

Wieviorka, Michel (1992): El espacio del racismo. Barcelona, Paidós.

Zwart (de), Frank (2005): “The Dilemma of Recognition: Administrative Categories and Cultural Diversity". En: Theory and Society, Vol. 34, n. 2, pp. 137-169; http:/ /www.jstor.org/stable/4501719 